KANSAS JOURNAL of MEDICINE

\section{Assessing Loneliness and Other Types of Emotional Distress among Practicing Physicians}

Samuel Ofei-Dodoo, Ph.D., MPA',

Christopher Ebberwein, Ph.D. ${ }^{1,2}$, Rick Kellerman, M.D. ${ }^{1}$

University of Kansas School of Medicine-Wichita, KS

${ }^{1}$ Department of Family and Community Medicine

${ }^{2}$ Family Medicine Residency Program, Wesley Medical Center

Received April 17, 2019; Accepted for publication. July 26, 2019; Published online Jan. 31, 2020

\section{ABSTRACT}

Introduction. Loneliness has been linked to clinician burnout and other types of emotional distress. Research assessing the prevalence of loneliness among physicians is growing. Little is known, however, about how loneliness relates to other types of emotional distress among practicing physicians. The objectives of the study were to determine the prevalence of loneliness, and to explore the relationship between loneliness, burnout, depressive symptoms, and suicidal ideation among active member physicians of the Medical Society of Sedgwick County (MSSC).

Methods. The study involved a convenience sample of 197 practicing physicians who were active members of the MSSC. The 3-item University of California, Los Angeles Loneliness Scale, the Abbreviated Maslach Burnout Inventory, and 2-item Primary Care Evaluation of Mental Disorders Patient Health Questionnaire were used to measure prevalence of loneliness, manifestations of burnout, and symptoms of depression, respectively.

Results. Using an email survey, 442 practicing physicians received an invitation to participate; 197 (44\%) completed the survey. The prevalence of loneliness was $43 \%$. Loneliness prevalence was associated positively with age $(\mathrm{p}=0.017)$ and more likely in those who reported manifestations of burnout $(\mathrm{p}<0.01)$ or screened positive for depression $(\mathrm{p}<0.01)$. Depression ( $\mathrm{OR}=2.24$; 95\% CI, 0.97-5.19) and emotional exhaustion (OR $=1.05$; 95\% CI, 0.39-2.84) were significantly associated with loneliness, including when adjusted for participants' sex, age, and duty hours.

Conclusion. Loneliness is prevalent among active member physicians of the Medical Society of Sedgwick County. Given that loneliness is associated with burnout and other emotional distress, there is an important need to understand its implications better.

\section{Kans J Med 2020;13:1-5. \\ INTRODUCTION}

Professionals across medical specialties are exploring the origins of burnout with a goal to understand better how to foster wellbeing and restore meaning in the work of medicine. ${ }^{1}$ Prior study has found a link between loneliness, depression, and health among older adults. ${ }^{2}$ Loneliness is experienced when individuals feel alone, and when they lack meaningful relationships regardless of the size of their social network. ${ }^{3,4}$ In studies that measure loneliness, participants often are asked if they feel alone, lack companionship, and wish to have more companionship. ${ }^{5}$ Loneliness has become a growing public health issue linked to a variety of health risks (e.g., high blood pressure, heart disease, obesity, a weakened immune system, anxiety, and depression). ${ }^{6}$ Because physicians are not immune to loneliness, it is worth exploring the link between loneliness and physician wellbeing.

Loneliness is a subjective distress and has been linked to burnout among internal medicine residents ${ }^{7}$ and has been cited as one of the major stressors of nurse managers. ${ }^{8}$ Increased support from family and friends reduces loneliness among resident physicians and subsequently reduces burnout. ${ }^{9}$ A study in the Harvard Business Review found that professionals such as doctors and lawyers have the highest rates of loneliness among all workers. ${ }^{10}$ Among practicing physicians, little is known about the link between loneliness and other types of emotional distress (i.e., burnout, depressive symptoms, and suicidal ideation). This study sought to explore the relationship between loneliness and other types of emotional distress among active member physicians of the Medical Society of Sedgwick County (MSSC) in Kansas.

\section{METHODS}

This study involved 197 practicing physicians who were active members of the MSSC. An email containing a link to a survey was sent to a convenience sample of 872 active members of the MSSC. Overall, 442 physicians opened at least one of the email invitations and were considered to have received the invitation to participate in the study. Data were gathered through anonymous surveys between July and August 2018. The data used for this analysis were part of a larger study on physician wellbeing. ${ }^{11}$ A sample size of 180 was calculated as necessary for adequate power $(>0.85)$ to detect a significant association among the variables with 1 degree of freedom, $\mathrm{p}<0.05$, and 0.5 effect size. ${ }^{12,13}$ The University of Kansas School of MedicineWichita Institutional Review Board granted exemption for the study.

Outcome Measures. The survey included items on loneliness, burnout, symptoms of depression, fatigue, suicidal ideation, and demographic information. The physicians' level of loneliness was assessed using the 3-item University of California, Los Angeles (UCLA) Loneliness Scale, a reliable and validated shortened screening tool to measure loneliness. ${ }^{14-16}$ Participants recorded their feeling of loneliness on a 3-point Likert scale: 1 = Hardly ever, 2 = Sometimes, and $3=$ Often, corresponding to scores of $1-3$ for each item. Scores for the three items were summed with a possible score of $3-9$. As a standard practice, ${ }^{15,17}$ physicians who had a total score of 6 or above were considered "lonely".

We used the Abbreviated Maslach Burnout Inventory (MBI9), a validated 9-item questionnaire to measure manifestations of burnout. ${ }^{18-20}$ The inventory measures professional burnout across three dimensions: emotional exhaustion, depersonalization, and perception of personal accomplishment. The physicians recorded their feelings on a 7-point Likert scale $(0=$ Never; $6=$ Every day $)$. Scores for the three items specific to each of the dimensions were summed with possible scores of 0 - 18. The scores within each dimension were grouped into low, intermediate, and high. Using established cutoffs, 
physicians who scored high on emotional exhaustion $(\geq 11)$ and/or depersonalization $(\geq 7)$ were considered to have at least one manifestation of burnout. $11,20,21$

A 2-item Primary Care Evaluation of Mental Disorders Patient Health Questionnaire was used to screen for symptoms of depression. The 2 -question screen has reported sensitivity of $96 \%$ and specificity of $57 \% .^{22,23}$ Suicidal ideation was measured by asking participants, "During the past 12 months, have you had thoughts of taking your own life?" This question has been used in previous studies involving physicians. ${ }^{24-26}$ The physicians' levels of fatigue during the prior week were assessed using a standardized linear analogue scale $(0=$ as bad as it can be; 10 = as good as it can be), similar to what is described by West et al. ${ }^{27}$ Lower scores on the scale indicated higher levels of fatigue. ${ }^{28,29}$

Statistical Analysis. Standard descriptive statistics were used to create a demographic profile and describe the prevalence of loneliness among the participants. Fisher's exact test was used to explore associations among the categorical variables. Generalized linear mixed models were used to calculate associations between the loneliness classification modeled as a binary outcome against a single fixed effect for independent variables (age, sex, depression, emotional exhaustion, depersonalization, personal accomplishment, fatigue, and suicidal intention). Adjusted odds ratios were estimated by modeling all significant independent variables against the loneliness classification, controlling for participants' sex, age, and duty hours. All analyses were performed with two-sided alpha of 0.05 .

\section{RESULTS}

There was a participation rate of $44.6 \%$ (197/442). Of the responding physicians, $53.8 \%$ were males; $89.3 \%$ were 35 years and older; $72.1 \%$ reported working more than 50 hours per week; $47.3 \%$ had been in practice for less than 20 years; and a plurality (24.4\%) was family physicians (Table 1). Analysis of the study sample compared to the overall MSSC population of active members showed a margin of error of $+/-6.2$ percentage points at a $95 \%$ confidence level, demonstrating that the demographic characteristics of this study generally represented the overall MSSC population of active members. ${ }^{30}$

The overall prevalence of loneliness was 43\% (84/197). The mean score on the UCLA 3-item Loneliness Scale was $5.2(\mathrm{SD}=1.9)$, with more than half of the physicians "sometimes" or "often" feeling lack of companionship, left out, or isolated from others (Figure 1). The prevalence of loneliness generally increased with age, as 5\% (4/84) of respondents aged $<35$ years reported loneliness compared to $37 \%$ $(31 / 84)$ of those aged $>55$ years $(p=0.017$; Figure 2$)$. Also, the physicians who experienced a greater feeling of loneliness were more likely to report at least one manifestation of burnout (64.8\% vs. 35.2\%, p < 0.01), screened positive for depression ( $66.3 \%$ vs. $33.7 \%, \mathrm{p}<0.01)$, experience a higher degree of fatigue $(64.8 \%$ vs. $35.2 \%, \mathrm{p}<0.01)$, and have thoughts of suicide ( $88.9 \%$ vs. $11.1 \%, p=0.012$ ).

As illustrated in Table 2, findings of the mixed model analyses indicated that there was a significant positive association between loneliness and depression $(\mathrm{p}<0.01)$. This association remained significant when adjusting for participants' sex, age, and duty hours (OR $=2.24$; 95\% CI, 0.97-5.19). Additionally, a high level of emotional
KANSAS JOURNAL of MEDIC INE

EMOTIONAL DISTRESS AMONG PRACTICING

\section{PHYSICIANS}

continued.

exhaustion correlated positively with prevalence of loneliness $(\mathrm{p}<$ 0.01 ), and this association remained significant when adjusting for sex, age, and duty hours (OR = 1.05; 95\% CI, 0.39-2.84).

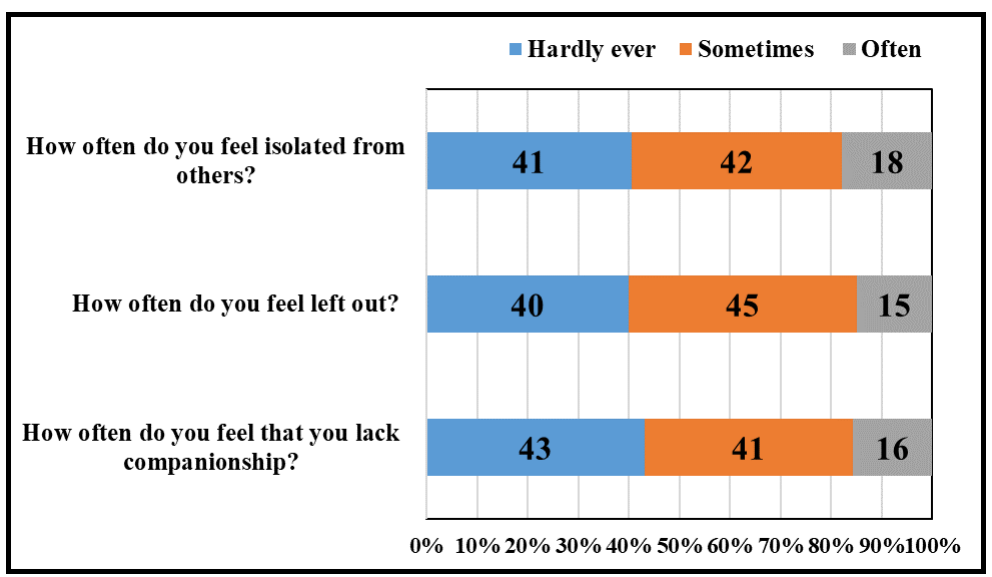

Figure 1. Percentage of physician responses to the 3-item UCLA Loneliness Scale $(N=197)$.

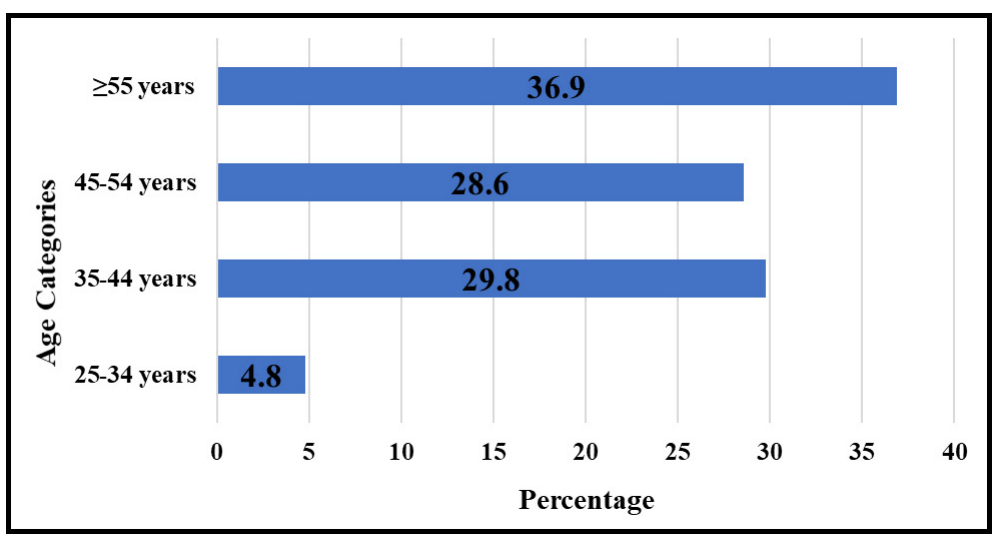

Figure 2. Prevalence of loneliness by age categories.

There was not a significant association between sexes $(\mathrm{p}=0.56)$ or duty hour fixed categories ( $p>0.05)$ and loneness score. Finally, there was no significant association between fatigue and loneliness score (p $=0.41)$, and with loneliness and suicidal ideation $(\mathrm{p}=0.23)$.

Given that a plurality of the respondents were family physicians ( $24.4 \%$ [48 of 197]), data on family medicine was compared with all other specialties combined. The prevalence of loneliness among family physicians ( $47.9 \%$ of 48 ) did not statistically differ from the prevalence among physicians of all specialties (44.4\% of 135 ; $\mathrm{p}>$ $0.05)$. 
KANSAS JOURNAL of MEDICINE EMOTIONAL DISTRESS AMONG PRACTICING PHYSICIANS

continued.

Table 1. Demographic characteristics of responding physicians. ${ }^{a}$

\begin{tabular}{|l|l|}
\hline Characteristics & Responders $(\mathrm{N}=197)$ \\
\hline Sex & $(\%)$
\end{tabular}

\begin{tabular}{|l|c|} 
Sex, No. $(\%)$ & $106(53.8)$ \\
\hline Male & $77(39.1)$ \\
\hline Female & $14(7.1)$ \\
\hline Missing &
\end{tabular}

\section{Age, No. (\%), years}

\begin{tabular}{|l|r|}
\hline $25-34$ & $8(4.3)$ \\
\hline $35-44$ & $54(29.3)$ \\
\hline $45-54$ & $46(25.0)$ \\
\hline $55-64$ & $54(29.3)$ \\
\hline$\geq 65$ & $22(12.0)$ \\
\hline Missing & $13(6.60)$ \\
\hline
\end{tabular}

\section{Years in Practice, No. $(\%)$}

\begin{tabular}{l|c}
\hline $1-9$ & $37(18.8)$ \\
\hline $10-19$ & $59(29.9)$ \\
\hline $20-29$ & $44(22.3)$ \\
\hline$\geq 30$ & $41(20.8)$ \\
\hline Missing & $16(8.1)$ \\
\hline
\end{tabular}

Hours worked per week, No. (\%)

\begin{tabular}{|l|c|}
\hline$<40$ & $17(8.6)$ \\
\hline $40-49$ & $38(19.3)$ \\
\hline $50-59$ & $59(29.9)$ \\
\hline $60-69$ & $39(19.8)$ \\
\hline $70-79$ & $13(6.6)$ \\
\hline$\geq 80$ & $17(8.6)$ \\
\hline Missing & $14(7.1)$ \\
\hline Specialty, No. (\%) & $5(2.5)$ \\
\hline Anesthesiology & $1(0.5)$ \\
\hline Dentistry & $1(0 . .5)$ \\
\hline Dermatology & $7(3.6)$ \\
\hline Emergency Medicine & $48(24.4)$ \\
\hline Family Medicine & $1(0.5)$ \\
\hline General Practice & $11(5.6)$ \\
\hline Hospitalist & $12(6.1)$ \\
\hline Internal Medicine sub-specialty & $12(6.1)$ \\
\hline Internal Medicine - general & $3(1.5)$ \\
\hline Neurology & $13(6.6)$ \\
\hline Obstetrics and Gynecology & $4(2.0)$ \\
\hline Ophthalmology & $4(2.0)$ \\
\hline Orthopedic Surgery & $6(3.0)$ \\
\hline Otolaryngology & \\
\hline Pathology & \\
\hline Pediatric sub-specialty & \\
\hline
\end{tabular}

\begin{tabular}{|l|c|}
\hline Characteristics & Responders (N = 197) \\
\hline Pediatrics - general & $13(6.6)$ \\
\hline Physical Medicine and Rehabilitation & $1(0.5)$ \\
\hline Psychiatry & $8(4.1)$ \\
\hline Radiation Oncology & $1(0.5)$ \\
\hline Radiology & $4(2.0)$ \\
\hline Surgery & $15(7.6)$ \\
\hline Other & $7(3.6)$ \\
\hline Missing & $14(7.1)$ \\
\hline
\end{tabular}

anformation is reported with permission from Ofei-Dodoo et al. ${ }^{11}$

Table 2. Unadjusted odds ratio for salient outcome measures and loneliness $(\mathrm{N}=197)$.

\begin{tabular}{|c|c|c|}
\hline Factors & Odds Ratio (95\% CI) & p value \\
\hline \multicolumn{3}{|l|}{ Sex } \\
\hline Male vs. Female & $1.28(0.61,2.69)$ & 0.56 \\
\hline \multicolumn{3}{|l|}{ Age } \\
\hline $25-34$ vs. $>64$ & $0.36(0.04,3.26)$ & 0.254 \\
\hline $35-44$ vs. $>64$ & $0.37(0.10,2.00)$ & 0.249 \\
\hline $45-54$ vs. $>64$ & $0.45(0.09,1.62)$ & 0.121 \\
\hline $55-64$ vs. $>64$ & $0.28(0.07,1.20)$ & 0.078 \\
\hline \multicolumn{3}{|l|}{ Weekly Duty Hours } \\
\hline $40-49$ vs. $<40$ & $0.90(0.16,4.95)$ & 0.817 \\
\hline $50-59$ vs. $<40$ & $1.15(0.29,4.60)$ & 0.926 \\
\hline $60-69$ vs. $<40$ & $1.71(0.45,6.49)$ & 0.575 \\
\hline $70-79$ vs. $<40$ & $1.02(0.26,4.02)$ & 0.822 \\
\hline$<79$ vs. $<40$ & $0.62(0.10,3.52)$ & 0.413 \\
\hline \multicolumn{3}{|l|}{ Depression } \\
\hline $\begin{array}{l}\text { Screened Positive vs. Screened } \\
\text { Negative }\end{array}$ & $2.24(0.97,5.19)$ & $<0.01$ \\
\hline \multicolumn{3}{|l|}{ Emotional Exhaustion } \\
\hline High vs. Intermediate & $3.68(1.21,11.16)$ & $<0.01$ \\
\hline High vs. Low & $1.99(0.78,5.10)$ & $<0.01$ \\
\hline \multicolumn{3}{|l|}{ Depersonalization } \\
\hline High vs. Intermediate & $1.82(0.69,4.78)$ & 0.55 \\
\hline High vs. Low & $1.26(0.42,3.77)$ & 0.21 \\
\hline \multicolumn{3}{|l|}{ Personal Accomplishment } \\
\hline Low vs. Intermediate & $1.14(0.43,3.02)$ & 0.081 \\
\hline Low vs. High & $0.87(0.28,2.67)$ & 0.31 \\
\hline \multicolumn{3}{|l|}{ Fatigue } \\
\hline High vs. Low & $0.51(0.23,1.11)$ & 0.41 \\
\hline \multicolumn{3}{|l|}{ Suicidal Ideation } \\
\hline Yes vs. No & $0.17(0.02,1.62)$ & 0.23 \\
\hline
\end{tabular}

$\mathrm{CI}=$ confidence interval 


\section{DISCUSSION}

Loneliness is defined as a lack of meaningful interpersonal relationships or interactions, ${ }^{3,4}$ and can affect people of all ages and backgrounds. This subjective feeling of loneliness depends more on the quality of relationships than on the number of people in one's social network. The findings of this exploratory study illustrated a connection between loneliness and other types of emotional distress among active member physicians of the MSSC. The prevalence of loneliness among the active member physicians of MSSC was $43 \%$ with older physicians reporting a significantly higher burden. This high rate of loneliness is not surprising given that workers with professional degrees, such as law and medicine, are the loneliest among all professional workers. ${ }^{10}$ The physicians who reported manifestations of burnout were at higher risk of feeling lonely. In addition to manifestations of burnout, positive loneliness screens were associated with depression and emotional exhaustion. These relationships between depression, emotional exhaustion, and loneliness were maintained even when adjusted for participants' sex, age, and duty hours.

Consistent with previous studies of internal medicine residents ${ }^{7}$ and nurse practitioners, ${ }^{8}$ our findings suggested that loneliness was associated with manifestations of burnout and symptoms of depression among practicing physicians. Response patterns appeared to be consistent with national survey results, ${ }^{26-28}$ with significant numbers of physicians reporting the aforementioned various signs of emotional distress.

A somewhat classic understanding of emotional exhaustion and depressed mood might explain the associations with loneliness found in this study. Emotional exhaustion is characterized by depletion of energy as a result of being overwhelmed by work; and depressed mood often is characterized by a lack of motivation or energy to engage with people and the tasks of everyday life. In other words, connecting meaningfully with others (a sort of antidote to loneliness) takes a particular level of effort and energy that might be missing when a person is emotionally exhausted or depressed. Further study of the link between loneliness and physician distress is warranted to help practicing physicians and health care systems thrive. Practical solutions could include fostering connections ${ }^{6,731-33}$ among physicians through socialization opportunities and/or support groups, as well as opportunities to build shared meaning ${ }^{34}$ (e.g., Balint Groups). These interventions have illustrated significant change in reducing loneliness scores ${ }^{35,36}$ and the high costs associated with the various manifestations of physician distress. ${ }^{37}$

This exploratory study was limited by a small sample size of active member physicians of the MSSC and non-probability convenience sampling, both of which reduced the generalizability of the findings. Additionally, as this study's results were limited to active member physicians of the MSSC, the findings may not be generalizable to physicians in other communities.

\section{CONCLUSIONS}

Our study suggested loneliness is prevalent among active member physicians of the MSSC. These findings suggested that loneliness is prevalent among physicians, and they contribute to the body of evidence indicating that loneliness is associated with burnout and other forms of emotional distress. Without attention to this issue, physicians and healthcare systems will continue to suffer the effects. Increased opportunities for meaningful connections with other physicians and increased opportunities for finding meaning in work should have a positive impact on the related issues of loneliness, emotional exhaustion, and depressed mood. Making Balint group participation more readily available (which may increase both connection and meaning) represents a practical step in searching for solutions to this widespread problem.

\section{ACKNOWLEDGEMENT}

The authors thank Mr. Phillip Brownlee of the Medical Society of Sedgwick County for the support and invaluable feedback on the manuscript.

\section{REFERENCES}

${ }^{1}$ Michel JB, Sangha DM, Erwin JP 3rd. Burnout among cardiologists. Am J Cardiol 2017; 119(6):938-940. PMID: 28065490.

2 Ong AD, Uchino BN, Wethington E. Loneliness and health in older adults: A mini-review and synthesis. Gerontology 2016; 62:443-449. PMID: 26539997.

3 Perlman D. European and Canadian studies of loneliness among seniors. Can J Aging 2004; 23(2):181-188. PMID: 15334817.

${ }^{4}$ Havens B, Hall M, Sylvestre G, Jivan T. Social isolation and loneliness: Differences between older rural and urban Manitobans. Can J Aging 2004; 23(2):129-140. PMID: 15334813.

5 Wenger GC, Burholt V. Changes in levels of social isolation and loneliness among older people in a rural area: A twenty-year longitudinal study. Can J Aging 2004; 23(2):115-127. PMID: 15334812.

6 Anderson GO, Thayer CE. Loneliness and social connections: A national survey of adults 45 and older. Washington, DC: AARP Research. 2018. Available at: https://doi.org/10.26419/res.00246.001. Accessed May 16, 2019.

7 Shapiro J, Zhang B, Warm EJ. Residency as a social network: Burnout, loneliness, and social network centrality. J Grad Med Educ 2015; 7(4):617623. PMID: 26692975.

8 Miyata A, Arai H, Suga S. Nurse managers stress and coping. Open J Nursing 2015; 5(11):957-964.

9 Rogers E, Polonijo AN, Carpiano RM. Getting by with a little help from friends and colleagues: Testing how residents' social support networks affect loneliness and burnout. Can Fam Physician 2016; 62(11):e677-e683. PMID: 28661887.

${ }^{10}$ Achor S, Kellerman GR, Reece A, Robichaux A. America's loneliest workers, according to research. Harvard Business Review. Available at: https://hbr.org/2018/03/americas-loneliest-workers-according-toresearch. Updated March 19, 2018. Accessed July 1, 2019.

${ }^{11}$ Ofei-Dodoo S, Kellerman R, Gilchrist K, Casey EM. Burnout and quality of life among active member physicians of the Medical Society of Sedgwick County. Kan J Med 2019; 12(2):33-39. PMID: 31191807.

${ }_{12}$ Australia and New Zealand Melanoma Trials Group Statistical Decision Tree. Power calculator for Chi-Square Test. Available at: https://www. anzmtg.org/stats/PowerCalculator/PowerChiSquare. Accessed Oct. 8, 2018.

${ }^{13}$ Kim HY. Statistical notes for clinical researchers: Chi-squared test and Fisher's exact test. Restor Dent Endod 2017; 42(2):152-155. PMID: 28503482.

${ }^{14}$ Hughes ME, Waite LJ, Hawkley LC, Cacioppo JT. A short scale for measuring loneliness in large surveys: Results from two population-based studies. Res Aging 2004; 26(6):655-672. PMID: 18504506.

${ }_{15}$ Steptoe A, Shankar A, Demakakos P, Wardle J. Social isolation, loneliness, and all-cause mortality in older men and women. Proc Natl Acad Sci US A 2013; 110(15):5797-5801. PMID: 23530191.

${ }^{16}$ Russell D, Peplau LA, Cutrona C. The revised UCLA Loneliness Scale: Concurrent and discriminant validity evidence. J Pers Soc Psychol 1980; 39(3):472-480. PMID: 7431205. 
${ }^{17}$ Mullen RA, Tong S, Sabo RT, et al. Loneliness in primary care patients: A prevalence study. Ann Fam Med 2019; 17(2):108-115. PMID: 30858253.

${ }_{18}$ McManus IC, Keeling A, Paice E. Stress, burnout and doctors' attitudes to work are determined by personality and learning style: A twelve year longitudinal study of UK medical graduates. BMC Med 2004; 2:29. PMID: 15317650.

19 Maslach C, Leiter MP. Early predictors of job burnout and engagement. J Appl Psychol 2008; 93(3):498-512. PMID: 18457483.

20 Sotile Center for Resilience. Maslach Burnout Inventory (Abbreviated) - MBI-9. Available at: https://www.nzgp-webdirectory.co.nz/site/nzgpwebdirectory2/Burnout-Abbreviated-General.pdf. Accessed on April 4, 2018.

${ }^{21}$ Maslach C, Jackson SF, Leiter MP. Maslach Burnout Inventory Manual. 3rd Ed. Palo Alto, CA: Consulting Psychologists Press, 1996.

${ }^{22}$ Whooley MA, Avins AL, Miranda J, Browner WS. Case-finding instruments for depression. Two questions are as good as many. J Gen Intern Med 1997; 12(7):439-445. PMID: 9229283.

${ }^{23}$ Spitzer RL, Williams JB, Kroenke K, et al. Utility of a new procedure for diagnosing mental disorders in primary care. The PRIME-MD 1000 study. JAMA 1994; 272(22):1749-1756. PMID: 7966923.

${ }^{24}$ Shanafelt TD, Balch CM, Dyrbye LN, et al. Special report: Suicidal ideation among American surgeons. Arch Surg 2011; 146(1):54-62. PMID: 21242446.

${ }^{25}$ van der Heijden F, Dillingh G, Bakker A, Prins J. Suicidal thoughts among medical residents with burnout. Arch Suicide Res 2008; 12(4):344-346. PMID: 18828037.

${ }^{26}$ Dyrbye LN, Thomas MR, Massie FS, et al. Burnout and suicidal ideation among U.S. medical students. Ann Intern Med 2008; 149(5):334-34l. PMID: 18765703.

27 West CP, Tan AD, Habermann TM, Sloan JA, Shanafelt TD. Association of resident fatigue and distress with perceived medical errors. JAMA 2009; 302(12):1294-1300. PMID: 19773564.

${ }^{28}$ Dyrby LN, West CP, Satele D, et al. Burnout among U.S. medical students, residents, and early career physicians relative to the general U.S. population. Acad Med 2014; 89(3):443-45l. PMID: 24448053.

${ }^{29}$ Sloan JA, Zhao X, Novotny PJ, et al. Relationship between deficits in overall quality of life and non-small-cell lung cancer survival. J Clin Oncol 2012; 30(13):1498-1504. PMID: 22454418.

30 Data Star, Inc. What Every Researcher Should Know About Statistical Significance. October 2008. Available at: http://www.surveystar.com/startips/oct2008.pdf. Accessed October 8, 2018.

${ }^{31}$ Kjeldmand D, Holmström I. Balint groups as a means to increase job satisfaction and prevent burnout among general practitioners. Ann Fam Med 2008; 6(2):138-145. PMID: 18332406.

32 Sandstrom GM, Dunn EW. Social interactions and well-being: The surprising power of weak ties. Pers Soc Psychol Bull 2014; 40(7):910-922. PMID: 24769739.

33 Engert V, Kok BE, Papassotiriou I, Chrousos GP, Singer T. Specific reduction in cortisol stress reactivity after social but not attention-based mental training. Sci Adv 2017; 3(10):el700495. PMID: 28983508.

34 Bonchek M. Purpose is good. Shared purpose is better. Harvard Business Review. Available at: https://hbr.org/2013/03/purpose-is-good-sharedpurpose. Updated March 14, 2013. Accessed July 1, 2019.

35 Masi CM, Chen HY, Hawkley LC, Cacioppo JT. A meta-analysis of interventions to reduce loneliness. Pers Soc Psychol Rev 2011; 15(3):219-266. PMID: 20716644.

36 Cacioppo S, Grippo AJ, London S, Goossens L, Cacioppo JT. Loneliness: Clinical import and interventions. Perspect Psychol Sci 2015; 10(2):238249. PMID: 25866548.

${ }^{37}$ Han S, Shanafelt TD, Sinsky CA, et al. Estimating the attributable cost of physician burnout in the United States. Ann Intern Med 2019. doi: 10.7326/ Mi8-1422. [Epub ahead of print]. PMID: 31132791. 